\title{
Development of a Brain Index for Dementia Diagnosis Using Quantitative EEG Analysis
}

\author{
Dongwook Han, PhD, $\mathrm{PT}^{1)}$ \\ 1) Department of Physical Therapy, College of Medical and Life Science, Silla University: 700 beon-gil, \\ 140 Baegyang-daero, Sasang-gu, Busan 617-736, Republic of Korea. TEL: +82 51-999-6238, \\ FAX: +82 51-999-5176
}

\begin{abstract}
Purpose] In this study, proposed and existing brain indexes derived from quantitative EEG analysis were compared in dementia cases and healthy subjects, to verify their clinical applicability in the diagnosis of dementia. [Subjects] The subjects of this study were 23 elderly women suffering from dementia and 18 elderly women without dementia, who consented to voluntary participation in this study after being informed of its purpose. [Method] There were two kinds of brain indexes used in this study. The first type was already in use and includes the attention (AT) index, the activation (AC) index, and the stress (ST) index. The second type of brain index, proposed by the author of this paper, comprises of the drowsiness (DS) index, the thinking (TK) index, and the complication (CP) index. [Result] There were significant differences between the AT index group and the TK index group in Fz, Fp1, and Fp2 of the prefrontal lobe. However, F3, F4, F7 and F8 of the frontal lobe, showed significant differences only in the AT index. For Pz, P3 and P4 of the parietal lobe, there were significant differences between the AT index group and the DS and TK index group. [Conclusion] The results show that a brain index for detecting dementia in a more accurate and objective way is needed, and that the development of a new brain index is feasible. Key words: Brain Index, Quantitative EEG, Brain activation
\end{abstract}

(This article was submitted Nov. 20, 2012, and was accepted Dec. 12, 2012)

\section{INTRODUCTION}

According to the 2007 report of Statistics Korea ${ }^{1)}$, 399,000 , or $8.3 \%$, of the entire elderly population of South Korea $(4,810,000)$, were suffering from dementia, and it was estimated that the number of the elderly suffering dementia would increase to 580,000 , or $9 \%$ by 2015 . The increasing numbers of elderly people with dementia has become a social issue in Korea. According to a 2008 report from the Korean Ministry of Health, Welfare and Family Affairs ${ }^{2)}$, there were 163,450 male and 257,936 female dementia patients in 2008 , accounting for $8.4 \%$ of the elderly population. The prevalence of dementia was predicted to increase to $9.1 \%$ in $2012,9.7 \%$ in 2020 , and $13.2 \%$ in 2050 . Since dementia is currently not curable after its onset, it is vital that dementia be prevented ${ }^{3}$. In light of how important it is to determine at an early stage the risk of developing dementia, various methods for detecting dementia are now under development. Among them, active research into brain waves, which can objectively estimate brain function, is progressing. Since a brain mapping function was recently added to the electroencephalogram (EEG) measurement system, not only is judgment based on visual aids made possible, but quantitative numeric values can now also be shown ${ }^{4,5}$. This quantitative electroencephalogram (qEEG) analysis can be used by non-specialists, and provides instant information from the EEG computer equipment ${ }^{6)}$.

E-mail:dwhan@silla.ac.kr
Hence, various studies using qEEG analysis have monitored the levels of brain function. Brain waves measured for qEEG analysis are generally Fourier transformed and then expressed as numeric values according to frequency band: $0-3 \mathrm{~Hz}$ is classified as delta waves, $4-7 \mathrm{~Hz}$ as theta waves, $8-13 \mathrm{~Hz}$ as alpha waves, and $14-30 \mathrm{~Hz}$ as beta waves ${ }^{7)}$. So far, most of the research that aims to diagnose dementia with the aid of EEG has resorted to qEEG analysis. For instance, Soininen et al. ${ }^{8)}$ examined whether there were differences in EEG results between elderly people who suffered Alzheimer's dementia and those who did not with the help of qEEG analysis. As a general result, a decrease of $\alpha$ waves and $\beta$ waves and an increase of $\theta$ waves and $\delta$ waves were found in those with Alzheimer's dementia. However, there has hardly been any research done to study the difference of brain waves in elderly subjects suffering dementia using the qEEG analysis method. Thus, the author of this study undertook research to ascertain if there is a difference in the brain waves of elderly women who suffer dementia and those who do not by means of qEEG analysis, hypothesizing that if cognition were good, $\theta$ waves would decrease while $\alpha$ waves would increase, and if cognition were bad then $\theta$ waves would be dominant while $\alpha$ waves would be inferior. The results show that in the frontal and parietal lobes, $\alpha$ waves were predominant in elderly women without dementia, while $\theta$ waves predominated in those with dementia. However, the results also show that $\alpha$ waves were as high in dementia patients as they were in women without dementia ${ }^{9}$. This result was somewhat different from those of Brenner et al. ${ }^{10)}$, who reported that visual EEG analysis, 
or qualitative EEG analysis, and analysis according to frequency band would both lead to similar results. This qEEG analysis showed that there was a possibility of error in diagnosing dementia when using only frequency band.

Hence, there is need for a method that is differentiated from qualitative EEG analysis and addresses the limitations of comparisons based on frequency band. Accordingly, the author hypothesized that a method comparing ratios of frequency bands would be more objective for checking the difference between subjects with dementia and those without than comparing only frequency bands. Prior to this, the author had investigated ${ }^{11)}$ whether there were any differences in qEEG between elderly women with dementia and those without dementia with the aid of the attention index (AT index), activation index (AC index), and stress index (ST index) as used by Han et al. ${ }^{12)}$, a team researching brain function using frequency band ratios. The results showed that the comparison of frequency bands ratios was more helpful in dementia diagnosis than a comparison of only frequency bands, since the AT index of elderly women without dementia was significantly lower than that in those with dementia, in all parts of both the frontal lobe and parietal lobe. Nevertheless, the AC index and ST index showed little difference between the two groups of elderly women, so it was considered that there was a need to determine the appropriate frequency band ratio for more precise dementia diagnosis.

Therefore, the first purpose of this study was to discover a more helpful and reliable frequency band ratio to diagnose dementia by comparing the conventionally used EEG analysis using the brain index to EEG analysis using new frequency band ratios. Secondly, this study investigated whether dementia could be accurately diagnosed with the help of the newly proposed brain indexes.

\section{SUBJECTS AND METHODS}

All 41 subjects who agreed to participate in the experiment had no record of cerebrovascular accident, stroke, or mental illness such as schizophrenia. None of the subjects had problems with independent daily living and all could communicate with the researchers of this study. For this study, the EEGs of elderly women both with dementia and without were measured and then classified according to frequency band through quantitative analysis. Then, the frequency band ratios were calculated and used as brain indexes.

EEG was measured by using an electroencephalographic monitor (Nihonkhoden, Inc., Japan). The attachment site of the electrodes was determined following the international standard of the 10-20 electrode method and included Fp1, Fp2, Fpz, F3, F4, F7, F8, Cz, C3, C4, T3, T4, T5, T6, Pz, $\mathrm{P} 3, \mathrm{P} 4, \mathrm{O} 1$ and $\mathrm{O} 2$, and the earlobe. For the measurement of EEG, the time constant was 0.3 seconds, sensitivity was $10 \mu \mathrm{V}$, and the cut-off frequency was $60 \mathrm{~Hz}$. First, the data of measured brain waves were Fourier transformed and categorized according to frequency band as follows: $\theta$ waves (4-7 Hz), $\alpha$ waves $(8-13 \mathrm{~Hz})$, SMR waves $(12-15 \mathrm{~Hz})$, low $\beta$ waves $(14-20 \mathrm{~Hz})$, high $\beta$ waves $(21 \mathrm{~Hz}-30 \mathrm{~Hz})$. The brain indexes of Han et al. ${ }^{12)}$ were then calculated. The attention index (AT index) is the ratio of $\theta$ waves to SMR waves, the activation index (AC index) is the ratio of low $\beta$ waves to $\alpha$ waves, and the stress index(ST index) is the ratio of high $\beta$ waves to $\alpha$ waves. The author then calculated new frequency band ratios to investigate their feasibility for dementia diagnosis.

The drowsiness index (DS index) was the ratio of $\theta$ waves versus $\alpha$ waves, the thinking index (TK index) was the ratio of low $\beta$ waves versus SMR waves, and the complication index (CP index) was the ratio of high $\beta$ waves versus SMR waves.

For data analysis, data were tested for normality using the Shapiro-Wilk test and normality was established. Thus differences in the brain indexes between the two groups were analyzed using the independent samples t-test as a parametric test. SPSSWIN (ver. 20.0) was used for data analysis and the significance level was chosen as $\alpha<0.05$.

\section{RESULTS}

The AT index of the elderly women suffering dementia was higher than that of those without dementia in all the areas of the frontal lobe-Fp1 $(p<0.01), F p 2(p<0.01)$, Fpz $(p<0.01), F 3(p<0.01), F 4(p<0.01), F 7(p<0.01)$, and F8 $(p<0.01)$, and the parietal lobe-Pz $(p<0.05), P 3(p<0.05)$, and P4 $(p<0.05)$. Furthermore, the AC index of elderly women without dementia was higher than that of those with dementia in the areas of Fp2 $(\mathrm{p}<0.05)$ in the frontal lobe and P3 $(\mathrm{p}<0.05)$, P4 $(\mathrm{p}<0.05)$ in the parietal lobe. In addition, the ST index of the women without dementia was higher than that of those with dementia in the Fp2 $(p<0.05)$ area of the frontal lobe and in the P4 $(\mathrm{p}<0.05)$ area of the parietal lobe (Table 1). However, among the newly proposed brain indexes, the DS index of the dementia sufferers was higher than that of the group without dementia in the F3 $(p<0.05)$ area of the frontal lobe and in all areas of the parietal lobe: $\mathrm{Pz}(\mathrm{p}<0.01), \mathrm{P} 3(\mathrm{p}<0.01)$, and P4 $(\mathrm{p}<0.01)$. Furthermore, the TK index of elderly women without dementia was higher than that of those with dementia in the areas of Fp1 $(p<0.05), F p 2(p<0.01)$, and F4 $(p<0.05)$ of the frontal lobe and in all areas of the parietal lobe: $\mathrm{Pz}(\mathrm{p}<0.05), \mathrm{P} 3$ $(\mathrm{p}<0.05)$, and P4 $(\mathrm{p}<0.05)$. The CP index of women without dementia was also higher than that of those with dementia, but only in the Fp2 $(\mathrm{p}<0.02)$ and F4 $(\mathrm{p}<0.05)$ areas of the frontal lobe (Table 2).

\section{DISCUSSION}

With the development of the Brain-Computer Interface (BCI) and monitoring technology, brain waves, which are electric signals occurring in the brain, can be utilized in various disciplines such as in all areas of rehabilitation, health screening, and other areas of medicine. Especially $\mathrm{BCI}$ is being applied to the clinical diagnosis of disorders such as epilepsy, Parkinson's disease, Alzheimer's disease, attention deficit hyperactivity disorder (ADHD) and other brain diseases ${ }^{13-15)}$. Since brain indexes calculated from frequency band ratios, are more objective than simple comparisons of frequency band, research using brain indexes is now underway in various disciplines. The brain indexes 
Table 1. The comparison of the existing brain indexes (unit: $\mu \mathrm{V}$ )

\begin{tabular}{|c|c|c|c|c|c|c|}
\hline & \multicolumn{2}{|c|}{ AT } & \multicolumn{2}{|c|}{$\mathrm{AC}$} & \multicolumn{2}{|c|}{ ST } \\
\hline & Normal & demented & Normal & demented & Normal & demented \\
\hline \multicolumn{7}{|c|}{ Prefrontal lobe } \\
\hline Fp1 & $1.43 \pm 0.84$ & $3.76 \pm 2.07 * *$ & $0.56 \pm 0.48$ & $0.35 \pm 0.20$ & $0.68 \pm 0.70$ & $0.40 \pm 0.42$ \\
\hline Fp2 & $1.51 \pm 0.96$ & $3.85 \pm 1.96^{* *}$ & $0.60 \pm 0.50$ & $0.32 \pm 0.17 *$ & $0.81 \pm 0.94$ & $0.30 \pm 0.24 *$ \\
\hline $\mathrm{Fz}$ & $1.62 \pm 0.92$ & $3.77 \pm 3.10^{* *}$ & $0.48 \pm 0.39$ & $0.33 \pm 0.20$ & $0.49 \pm 0.59$ & $0.24 \pm 0.28$ \\
\hline \multicolumn{7}{|c|}{ Frontal lobe } \\
\hline F3 & $1.32 \pm 0.74$ & $3.18 \pm 2.67 * *$ & $0.54 \pm 0.44$ & $0.37 \pm 0.22$ & $0.54 \pm 0.63$ & $0.30 \pm 0.31$ \\
\hline $\mathrm{F} 4$ & $1.41 \pm 0.77$ & $2.92 \pm 1.91 * *$ & $0.59 \pm 0.50$ & $0.36 \pm 0.22$ & $0.63 \pm 0.70$ & $0.27 \pm 0.28$ \\
\hline F7 & $1.37 \pm 0.85$ & $3.22 \pm 2.17 * *$ & $0.52 \pm 0.47$ & $0.39 \pm 0.33$ & $0.51 \pm 0.52$ & $0.39 \pm 0.49$ \\
\hline F8 & $1.27 \pm 0.82$ & $2.93 \pm 2.15^{* *}$ & $0.51 \pm 0.38$ & $0.39 \pm 0.25$ & $0.55 \pm 0.55$ & $0.38 \pm 0.38$ \\
\hline \multicolumn{7}{|c|}{ Parietal lobe } \\
\hline $\mathrm{Pz}$ & $1.37 \pm 1.23$ & $2.63 \pm 1.94 *$ & $0.73 \pm 1.12$ & $0.22 \pm 0.21$ & $0.48 \pm 0.68$ & $0.14 \pm 0.19$ \\
\hline P3 & $1.19 \pm 1.25$ & $2.32 \pm 1.70^{*}$ & $0.53 \pm 0.53$ & $0.22 \pm 0.17 *$ & $0.41 \pm 0.60$ & $0.14 \pm 0.19$ \\
\hline P4 & $1.23 \pm 1.06$ & $2.70 \pm 2.34 *$ & $0.59 \pm 0.60$ & $0.19 \pm 0.19 *$ & $0.49 \pm 0.63$ & $0.13 \pm 0.19 *$ \\
\hline
\end{tabular}

Mean \pm SD ** $: \mathrm{p}<0.01, *$ : $<<0.05$

AT: Attention Index, AC: Activation Index, ST: Stress Index

Table 2. The comparison of the new brain indexes (unit: $\mu \mathrm{V}$ )

\begin{tabular}{|c|c|c|c|c|c|c|}
\hline & \multicolumn{2}{|c|}{ DS } & \multicolumn{2}{|c|}{ TK } & \multicolumn{2}{|c|}{$\mathrm{CP}$} \\
\hline & Normal & demented & Normal & demented & Normal & demented \\
\hline \multicolumn{7}{|c|}{ Prefrontal lobe } \\
\hline Fp1 & $0.36 \pm 0.28$ & $0.43 \pm 0.28$ & $2.18 \pm 0.74$ & $1.75 \pm 0.61^{*}$ & $2.33 \pm 1.07$ & $2.09 \pm 2.40$ \\
\hline $\mathrm{Fp} 2$ & $0.37 \pm 0.32$ & $0.45 \pm 0.18$ & $2.28 \pm 0.82$ & $1.67 \pm 0.43^{* *}$ & $2.51 \pm 1.56$ & $1.56 \pm 0.93^{*}$ \\
\hline $\mathrm{Fz}$ & $0.37 \pm 0.31$ & $0.53 \pm 0.25$ & $2.06 \pm 0.78$ & $1.69 \pm 0.58$ & $1.72 \pm 1.10$ & $1.16 \pm 0.86$ \\
\hline \multicolumn{7}{|c|}{ Frontal lobe } \\
\hline F3 & $0.34 \pm 0.29$ & $0.53 \pm 0.25^{*}$ & $2.09 \pm 0.71$ & $1.80 \pm 0.59$ & $1.82 \pm 1.01$ & $1.47 \pm 1.18$ \\
\hline F4 & $0.36 \pm 0.30$ & $0.51 \pm 0.19$ & $2.24 \pm 0.81$ & $1.73 \pm 0.53 *$ & $2.03 \pm 1.22$ & $1.28 \pm 0.75^{*}$ \\
\hline F7 & $0.32 \pm 0.24$ & $0.36 \pm 0.26$ & $1.96 \pm 0.63$ & $1.77 \pm 0.58$ & $1.79 \pm 0.85$ & $1.51 \pm 0.96$ \\
\hline F8 & $0.32 \pm 0.25$ & $0.35 \pm 0.24$ & $2.04 \pm 0.70$ & $1.77 \pm 0.53$ & $1.90 \pm 0.97$ & $1.56 \pm 1.03$ \\
\hline \multicolumn{7}{|c|}{ Parietal lobe } \\
\hline $\mathrm{Pz}$ & $0.30 \pm 0.18$ & $4.49 \pm 3.57^{* *}$ & $2.28 \pm 1.83$ & $1.28 \pm 0.34^{*}$ & $1.40 \pm 1.25$ & $0.76 \pm 0.40$ \\
\hline P3 & $0.24 \pm 0.18$ & $4.60 \pm 3.34 * *$ & $1.99 \pm 1.08$ & $1.29 \pm 0.37 *$ & $1.29 \pm 1.08$ & $0.80 \pm 0.42$ \\
\hline P4 & $0.28 \pm 0.20$ & $5.15 \pm 4.41^{* *}$ & $1.86 \pm 0.81$ & $1.33 \pm 0.36^{*}$ & $1.36 \pm 1.13$ & $0.78 \pm 0.47$ \\
\hline
\end{tabular}

Mean \pm SD $\quad * *: \mathrm{p}<0.01, \quad *: \mathrm{p}<0.05$

DS: Drowsiness Index, TK: Thinking Index， CP: Complication Index

generally used are the AT index, AC index, and ST index, of Han et al. ${ }^{12)}$ Park ${ }^{16)}$ stated that the AT index is the value of $\theta$ waves divided by SMR waves, and that the lower the figure is, the more alert the brain is. The AC index is obtained by dividing low frequency $\beta$ waves with $\alpha$ waves, and a high value indicates a highly activated condition of the brain. The ST index is the value of high frequency $\beta$ waves divided by $\alpha$ waves; the higher its value, the more stressed and tired the brain is. Thus, in the case of the elderly, it was speculated that the AT index would be high, but that the AC and ST indexes would be low. The results of this study support this hypothesis, showing that the AT index of the elderly women with dementia was higher than that of the elderly women without dementia in all parts of the frontal lobe (Fp1, Fp2, Fpz, F3, F4, F7 and F8) and in all areas of the parietal lobe (Pz, P3 and P4) as well.

The AC index was shown to be significantly higher in the group without dementia than in the group with dementia at Fp2 of the frontal lobe, and P3 and P4 of the parietal lobe. The ST index of the dementia group was also significantly higher than that of the dementia group at $\mathrm{Fp} 2$ of the frontal lobe, and $\mathrm{p} 4$ of the parietal lobe. This result was similar to that of the 2011 experiment done by the author of this study ${ }^{11)}$, and it might indicate that diagnosis for dementia with the help of the brain index is possible and feasible. However, since there was only one brain index, the AT index, that showed a clear discrepancy between the two groups, the necessity for creating new brain indexes to reduce the possibility of erroneous diagnosis of dementia became clear. Therefore, the author proposed the drowsiness 
index (DS index), the thinking index (TK index) and the complication index (CP index), and investigated their feasibility in the diagnosis of dementia. The DS index showed significant statistical difference in all parts of the parietal lobe, Pz, P3, and P4, but in only one part of the frontal lobe, F3. The TK index showed significant differences in some parts of the frontal lobe, Fp1, Fp2 and F4 and significant differences in all areas of the parietal lobe: Pz, P3, and P4. The CP index also showed significant differences in Fp2 and F4 of the frontal lobe, but no difference in any part of the parietal lobe.

These results indicated that the AT and TK indexes discriminate between women with dementia and those without at Fz, Fp1 and Fp2 of the prefrontal lobe. Furthermore, the AT index successfully discriminated between the two groups of women at F3, F4, F7, and F8 of the frontal lobe. On the other hand, the AT, DS and TK indexes effectively discriminated between the two groups in the parietal lobe parts of Pz, P3 and P4. These results indicate that using brain indexes for diagnosing dementia gives better results than simple comparison of frequency bands. Furthermore, these results show that it is possible to create new brain indexes for more accurate and objective diagnoses of dementia.

\section{REFERENCES}

1) Korean national statistical office: Population projections for Korea, 2007.

2) Korean ministry of health, welfare and family affairs: nationwide study on the prevalence of dementia in Korean elders, 2008

3) Scarmeas N, Luchsinger JA, Schupf N, et al.: Physical activity, diet, and risk of Alzheimer disease. JAMA, 2009, 302: 627-637. [Medline] [CrossRef]

4) Massetani R, Strata G, Galli R, et al.: Alteration of cardiac function in patients with temporal lobe epilepsy: different roles of EEG-ECG monitoring and spectral analysis of RR variability. Epilepsia, 1997, 38: 363-369. [Medline] [CrossRef]

5) McKeown MJ, Young GB: Digital conversion of paper electroencephalograms using a hand scanner. J Clin Neurophysiol, 1997, 14: 406-413. [Medline] [CrossRef]

6) Park SY, Ko MM: A Study on SEEG (Statistical EEG Analysis). J Korean Data Anal Soc, 2008, 10: 1313-1325.

7) Ko BJ, Shim JY: Effects of brain development program for the youth on changes in EEG by brain areas depending on level of concentration and mental power. Youth Facility Environ, 2012, 10: 3-17.

8) Soininen H, Partanen J, Laulumaa V, et al.: Longitudinal EEG spectral analysis in elderly stage of Alzheimers disease. Electroencephalogr Clin Neurophysiol, 1989, 72: 290-297. [Medline] [CrossRef]

9) Han DW, Ha MS: Quantitative analysis on the difference of EEG power and coherence between normal elderly women and demented elderly women with closed their eyes. J Korean Data Anal Soc, 2008, 10: 3077-3089.

10) Brenner RP, Reynolds CF, Ulrich RP: Diagnostic efficacy of computerized spectral versus visual EEG analysis in elderly normal, demented and depressed subjects. Electroencephalogr Clin Neurophysiol, 1988, 69: 110117. [Medline] [CrossRef]

11) Ha MS, Han DW: On the interaction between electroencephalogram bands of normal elderly women and demented elderly women. J Phys Ther Sci, 2011, 23: 233-235. [CrossRef]

12) Han YS, Chae MS, Park BW, et al.: Prefrontal brain waves of cancer patients using brain-computer-interface. J KISS, 2008, 35: 169-178

13) Shin SC, Ryu CS, Song YS, et al.: EEC-based Subjects' response time detection for brain - computer - interface. J KIISE Softw Appl, 2002, 29: $837-850$.

14) Shin JH, Seo EM: Entertaining technical development trends based on BCI. J Korean Inst Electron Engineers, 2007, 34: 679-690.

15) Eum TW, Kim ES: EEG-based brain computer interface technology. Korea Inf Sci Soc Rev, 2004, 22: 5-19.

16) Park BW: A study on optimization of brain function using correlation between EEG band. Proc Korean Jungshin Sci Soc, 2004, 21: 103-108. 